Algebraic $8 \mathcal{G}$ Geometric $\mathcal{T}$ opology

Volume 4 (2004) 757-780

Published: 11 September 2004

ATG

\title{
The braid groups of the projective plane
}

\author{
Daciberg Lima Gonçalves \\ JOHN GUASCHI
}

\begin{abstract}
Let $B_{n}\left(\mathbb{R} P^{2}\right)$ (respectively $P_{n}\left(\mathbb{R} P^{2}\right)$ ) denote the braid group (respectively pure braid group) on $n$ strings of the real projective plane $\mathbb{R} P^{2}$. In this paper we study these braid groups, in particular the associated pure braid group short exact sequence of Fadell and Neuwirth, their torsion elements and the roots of the 'full twist' braid. Our main results may be summarised as follows: first, the pure braid group short exact sequence $1 \longrightarrow P_{m-n}\left(\mathbb{R} P^{2} \backslash\left\{x_{1}, \ldots, x_{n}\right\}\right) \longrightarrow P_{m}\left(\mathbb{R} P^{2}\right) \longrightarrow P_{n}\left(\mathbb{R} P^{2}\right) \longrightarrow 1$ does not split if $m \geq 4$ and $n=2,3$. Now let $n \geq 2$. Then in $B_{n}\left(\mathbb{R} P^{2}\right)$, there is a $k$-torsion element if and only if $k$ divides either $4 n$ or $4(n-1)$. Finally, the full twist braid has a $k^{\text {th }}$ root if and only if $k$ divides either $2 n$ or $2(n-1)$.
\end{abstract}

AMS Classification Primary: 20F36, 55R80; Secondary: 55Q52, 20F05

Keywords Braid group, configuration space, torsion, Fadell-Neuwirth short exact sequence

\section{Introduction}

Braid groups of the plane $\mathbb{E}^{2}$ were defined by Artin in 1925 A1, and further studied in [A2, A3. They were later generalised using the following definition due to Fox [FoN]. Let $M$ be a compact, connected surface, and let $n \in \mathbb{N}$. We denote the set of all ordered $n$-tuples of distinct points of $M$, known as the $n^{\text {th }}$ configuration space of $M$, by:

$$
F_{n}(M)=\left\{\left(p_{1}, \ldots, p_{n}\right) \mid p_{i} \in M \text { and } p_{i} \neq p_{j} \text { if } i \neq j\right\} .
$$

The configuration spaces $F_{n}(M)$ play an important rôle in several branches of mathematics and have been extensively studied, see [CG] for example.

The symmetric group $S_{n}$ on $n$ letters acts freely on $F_{n}(M)$ by permuting coordinates. The corresponding quotient will be denoted by $D_{n}(M)$. Notice that $F_{n}(M)$ is a regular covering of $D_{n}(M)$. The $n^{\text {th }}$ pure braid group $P_{n}(M)$ (respectively the $n^{\text {th }}$ braid group $B_{n}(M)$ ) is defined to be the fundamental 
group of $F_{n}(M)$ (respectively of $D_{n}(M)$ ). If $m>n$ are positive integers, then we may define a homomorphism $p_{*}: P_{m}(M) \longrightarrow P_{n}(M)$ induced by the projection $p: F_{m}(M) \longrightarrow F_{n}(M)$ defined by $p\left(\left(x_{1}, \ldots, x_{m}\right)\right)=\left(x_{1}, \ldots, x_{n}\right)$. Representing $P_{m}(M)$ geometrically as a collection of $m$ strings, $p_{*}$ corresponds to forgetting the last $(m-n)$ strings.

We adopt the convention that, unless explicitly stated, all homomorphisms $P_{m}(M) \longrightarrow P_{n}(M)$ in the text will be this one.

If $M$ is without boundary, Fadell and Neuwirth study the map $p$, and show [FaN Theorem 3] that it is a locally trivial fibration. The fibre over a point $\left(x_{1}, \ldots, x_{n}\right)$ of the base space is $F_{m-n}\left(M \backslash\left\{x_{1}, \ldots, x_{n}\right\}\right)$ which we consider to be a subspace of the total space via the map $i: F_{m-n}\left(M \backslash\left\{x_{1}, \ldots, x_{n}\right\}\right) \longrightarrow$ $F_{m}(M)$ defined by $i\left(\left(y_{1}, \ldots, y_{m-n}\right)\right)=\left(x_{1}, \ldots, x_{n}, y_{1}, \ldots, y_{m-n}\right)$. Applying the associated long exact homotopy sequence, we obtain the generalised pure braid group short exact sequence of Fadell and Neuwirth:

$$
1 \longrightarrow P_{m-n}\left(M \backslash\left\{x_{1}, \ldots, x_{n}\right\}\right) \stackrel{i_{*}}{\longrightarrow} P_{m}(M) \stackrel{p_{*}}{\longrightarrow} P_{n}(M) \longrightarrow 1,
$$

where $n \geq 3$ if $M=\mathbb{S}^{2}\left[\mathrm{Fa}\right.$, [FV], $n \geq 2$ if $M=\mathbb{R} P^{2}[\mathrm{VB}$, and $n \geq 1$ otherwise [FaN], and where $i_{*}$ and $p_{*}$ are the homomorphisms induced by the maps $i$ and $p$ respectively. The sequence also exists in the case where $M$ is the 2 -disc $\mathbb{D}^{2}$.

This short exact sequence has been widely studied, notably in recent work in relation to the structure of the mapping class groups $[\mathrm{PR}$. An interesting question is that of whether the sequence (PBS) splits. If $M$ is a $K(\pi, 1)$ then the existence of a section for $p_{*}$ is equivalent to that of a cross-section for the fibration. In $\mathrm{A} 2$, Artin showed that if $M=\mathbb{D}^{2}$ then the sequence (PBS) splits for all $n \in \mathbb{N}$. He used this result to solve the word problem in $B_{n}\left(\mathbb{D}^{2}\right)$ by showing that $P_{n}\left(\mathbb{D}^{2}\right)$ may be expressed as a repeated semi-direct product of free groups. Since then, the splitting problem has been studied for other surfaces besides the plane. In the case $M=\mathbb{S}^{2}$, it was known that there exists a section on the geometric level for all $n \geq 3[\mathrm{Fa}$. If $M$ is the 2-torus then for all $n \geq 1$, Birman exhibits an explicit algebraic section for the sequence (PBS) Bi]. However, for compact orientable surfaces without boundary of genus $g \geq 2$, she poses the question of whether the short exact sequence (PBS) splits. The authors of this paper have recently resolved this question, the answer being positive if and only if $n=1$ [GG1]. We will treat the case of non-orientable surfaces of genus at least two in a forthcoming paper [GG3].

In this paper we shall study the braid groups of $\mathbb{R} P^{2}$, in particular the splitting of the sequence ( $\mathbf{P B S})$, and the existence of a section for the fibration $p$. 
These groups were first studied by Van Buskirk $\mathrm{VB}$, and more recently by Wang Wa. Van Buskirk showed that $P_{1}\left(\mathbb{R} P^{2}\right)=B_{1}\left(\mathbb{R} P^{2}\right) \cong \mathbb{Z}_{2}, P_{2}\left(\mathbb{R} P^{2}\right)$ is isomorphic to the quaternion group $Q_{8}, B_{2}\left(\mathbb{R} P^{2}\right)$ is a dicyclic group of order 16 , and for $n>2, P_{n}\left(\mathbb{R} P^{2}\right)$ and $B_{n}\left(\mathbb{R} P^{2}\right)$ are infinite. He also proved that these groups have elements of finite order (including one of order $2 n$ ), such elements later being characterised by Murasugi $[\mathrm{M}]$. With respect to the splitting problem, Van Buskirk showed that for $n \geq 2$, neither the fibration $p: F_{n}\left(\mathbb{R} P^{2}\right) \longrightarrow F_{1}\left(\mathbb{R} P^{2}\right)$ nor the homomorphism $p_{*}: P_{n}\left(\mathbb{R} P^{2}\right) \longrightarrow P_{1}\left(\mathbb{R} P^{2}\right)$ admit a cross-section, but that the fibration $p: F_{3}\left(\mathbb{R} P^{2}\right) \longrightarrow F_{2}\left(\mathbb{R} P^{2}\right)$ admits a cross-section (and hence so does the corresponding homomorphism). In order to understand better the configuration space $F_{n}\left(\mathbb{R} P^{2}\right), n \in \mathbb{N}$, in Proposition 6 we determine the homotopy type of its universal covering space. From this, we may deduce its $i^{\text {th }}$ homotopy groups, $i \geq 2$ (see Section 2):

Proposition 1 Let $n \in \mathbb{N}$, and let $i \geq 2$.

(1) If $n=1$ then $\pi_{i}\left(F_{1}\left(\mathbb{R} P^{2}\right)\right) \cong \pi_{i}\left(\mathbb{S}^{2}\right)$.

(2) If $n \geq 2$ then $\pi_{i}\left(F_{n}\left(\mathbb{R} P^{2}\right)\right) \cong \pi_{i}\left(\mathbb{S}^{3}\right)$.

We then show that for $m \geq 4$ and $n=2,3$, neither the fibration nor the short exact sequence ( $\overline{\text { PBS }}$ ) admit a section. More precisely:

Theorem 2 Let $m \geq 4$. Then:

(1) the fibration $p: F_{m}\left(\mathbb{R} P^{2}\right) \longrightarrow F_{2}\left(\mathbb{R} P^{2}\right)$ does not admit a cross-section;

(2) the fibration $p: F_{m}\left(\mathbb{R} P^{2}\right) \longrightarrow F_{3}\left(\mathbb{R} P^{2}\right)$ does not admit a cross-section.

Theorem 3 For $n=2,3$ and for all $m \geq 4$, the Fadell-Neuwirth pure braid group short exact sequence :

$$
1 \longrightarrow P_{m-n}\left(\mathbb{R} P^{2} \backslash\left\{x_{1}, \ldots, x_{n}\right\}\right) \stackrel{i_{*}}{\longrightarrow} P_{m}\left(\mathbb{R} P^{2}\right) \stackrel{p_{*}}{\longrightarrow} P_{n}\left(\mathbb{R} P^{2}\right) \longrightarrow 1
$$

does not split.

Also in Section 2] we give an explicit algebraic section in the case $m=3$ and $n=2$, which we use in Section 3 to study further the structure of $P_{3}\left(\mathbb{R} P^{2}\right)$, notably its subgroups abstractly isomorphic to $P_{2}\left(\mathbb{R} P^{2}\right)$.

Let $\mathbb{D}^{2} \subseteq \mathbb{R} P^{2}$ be a topological disc. This inclusion induces a (non-injective) homomorphism $\iota: B_{n}\left(\mathbb{D}^{2}\right) \longrightarrow B_{n}\left(\mathbb{R} P^{2}\right)$. Let $\sigma_{1}, \ldots, \sigma_{n-1}$ be a standard set of generators of $B_{n}\left(\mathbb{D}^{2}\right)$, and let:

$$
\Delta_{n}=\left(\sigma_{1} \cdots \sigma_{n-1}\right)^{n},
$$


be the full twist braid of $B_{n}\left(\mathbb{D}^{2}\right)$. By convention, we set $\Delta_{1}=1$. The braid $\iota\left(\Delta_{n}\right)$ which for $n \geq 2$ is of order 2 and generates the centre $Z\left(B_{n}\left(\mathbb{R} P^{2}\right)\right)$ of $B_{n}\left(\mathbb{R} P^{2}\right)\left[\mathrm{VB}\right.$, shall also be denoted by $\Delta_{n}$, and will be called the full twist of $B_{n}\left(\mathbb{R} P^{2}\right)$. If $k \in \mathbb{N}$, then we shall say that $\alpha \in B_{n}(M)$ is a $k^{\text {th }}$ root of $\Delta_{n}$ if $\alpha^{k}=\Delta_{n}$ and $\alpha^{j} \neq \Delta_{n}$ for all $1 \leq j<k$. As we previously mentioned, the torsion elements of $B_{n}\left(\mathbb{R} P^{2}\right)$, as well as the roots of the full twist were characterised by Murasugi. However, in both cases, the orders of the elements are not completely clear, even for $P_{n}\left(\mathbb{R} P^{2}\right)$. In Section 3, we study the torsion as well as the roots of $\Delta_{n}$ in $P_{n}\left(\mathbb{R} P^{2}\right)$ using methods different to those of Murasugi, and prove that for all $n \geq 2$ the torsion of $P_{n}\left(\mathbb{R} P^{2}\right)$ is precisely 2 and 4 (see Corollary 19). In Section 4, we do the same for $B_{n}\left(\mathbb{R} P^{2}\right)$ :

Theorem 4 Let $n \geq 2$. Then $B_{n}\left(\mathbb{R} P^{2}\right)$ has an element of order $\ell$ if and only if $\ell$ divides either $4 n$ or $4(n-1)$.

From Proposition 23 (see also Section 41), $\Delta_{n}$ is the unique element of $B_{n}\left(\mathbb{R} P^{2}\right)$ of order 2. It thus follows that:

Theorem 5 Let $n \geq 2$. Then the full twist $\Delta_{n}$ has a $k^{\text {th }}$ root in $B_{n}\left(\mathbb{R} P^{2}\right)$ if and only if $k$ divides either $2 n$ or $2(n-1)$.

Acknowledgements This work began during the visit of the second author to the Departmento de Matemática do IME-Universidade de São Paulo during the period $7^{\text {th }}$ July $-3^{\text {rd }}$ August 2002. The visit was supported by the international Cooperation Capes/Cofecub project number 364/01.

\section{The splitting problem for $\mathbb{R} P^{2}$}

In this section, we recall, reformulate and generalise some results obtained in $\left[\mathrm{Fa}, \mathrm{VB}\right.$ ] concerning the configuration spaces $F_{n}\left(\mathbb{R} P^{2}\right)$ of the projective plane. If $n \geq 2$, we show that the universal covering of the configuration spaces $F_{n}\left(\mathbb{R} P^{2}\right)$ has the homotopy type of the 3 -sphere $\mathbb{S}^{3}$. We then prove that if $n \geq 2$, the problem of finding a cross-section for the fibrations $F_{m}\left(\mathbb{R} P^{2}\right) \stackrel{p}{\longrightarrow}$ $F_{n}\left(\mathbb{R} P^{2}\right)$ introduced in Section 1 is equivalent to that of the splitting of the corresponding pure braid group short exact sequence. Finally, we prove Theorems 2 and 3, that is, if $n=2,3$ and $m \geq 4$ then the fibration $F_{m}\left(\mathbb{R} P^{2}\right) \stackrel{p}{\longrightarrow} F_{n}\left(\mathbb{R} P^{2}\right)$ does not admit a cross-section, with a similar conclusion for the splitting of the short exact sequence (PBS). 


\subsection{The configuration spaces $F_{n}\left(\mathbb{R} P^{2}\right)$ and a description of their homotopy groups}

We first determine the homotopy type of the universal covering of the configuration spaces $F_{n}\left(\mathbb{R} P^{2}\right)$ and discuss their fundamental groups. Partial results in this direction were obtained in $\mathrm{Fa}, \mathrm{VB}$.

\section{Proposition 6}

(1) Let $m>n \geq 2$. Then for each $i>1$, the projection $p: F_{m}\left(\mathbb{R} P^{2}\right) \longrightarrow$ $F_{n}\left(\mathbb{R} P^{2}\right)$ onto the first $n$ coordinates induces an isomorphism of the corresponding $i^{\text {th }}$ homotopy groups.

(2) For $n=1$, the universal covering of $F_{n}\left(\mathbb{R} P^{2}\right)$ is the sphere $\mathbb{S}^{2}$. The fundamental group of $F_{1}\left(\mathbb{R} P^{2}\right)$ is $\mathbb{Z}_{2}$.

(3) For $n=2$, the fundamental group of $F_{n}\left(\mathbb{R} P^{2}\right)$ is isomorphic to the quaternion group $Q_{8}$ of order 8.

(4) For $n \geq 3$, the fundamental group of $F_{n}\left(\mathbb{R} P^{2}\right)$ is infinite.

(5) For $n \geq 2$, the homotopy type of the universal covering of $F_{n}\left(\mathbb{R} P^{2}\right)$ is that of the 3 -sphere $\mathbb{S}^{3}$.

(6) The orbit space arising from the standard action of $Q_{8}$ on $\mathbb{S}^{3}$ has the same homotopy type as $F_{2}\left(\mathbb{R} P^{2}\right)$.

Notice that Proposition [ implies Proposition 1

Proof of Proposition [6 To prove part (11), let $m>n \geq 2$. For $i>1$, consider the long exact sequence in homotopy associated to the (locally trivial) fibration $p: F_{m}\left(\mathbb{R} P^{2}\right) \longrightarrow F_{n}\left(\mathbb{R} P^{2}\right)$ given by $p\left(\left(x_{1}, \ldots, x_{m}\right)\right)=\left(x_{1}, \ldots, x_{n}\right)$ :

$$
\begin{aligned}
& \cdots \longrightarrow \pi_{i}(F) \longrightarrow \pi_{i}\left(F_{m}\left(\mathbb{R} P^{2}\right)\right) \longrightarrow \pi_{i}\left(F_{n}\left(\mathbb{R} P^{2}\right)\right) \longrightarrow \pi_{i-1}(F) \longrightarrow \cdots \\
& \cdots \longrightarrow \pi_{2}(F) \longrightarrow \pi_{2}\left(F_{m}\left(\mathbb{R} P^{2}\right)\right) \longrightarrow \pi_{2}\left(F_{n}\left(\mathbb{R} P^{2}\right)\right) \longrightarrow \pi_{1}(F) \longrightarrow \cdots
\end{aligned}
$$

where $F=F_{m-n}\left(\mathbb{R} P^{2} \backslash\left\{x_{1}, \ldots, x_{n}\right\}\right)$ is the fibre over $\left(x_{1}, \ldots, x_{n}\right) \in F_{n}\left(\mathbb{R} P^{2}\right)$. Since $F$ is an aspherical space [FaN], and $\pi_{2}\left(F_{q}\left(\mathbb{R} P^{2}\right)\right)=0$ for all $q \geq 2[\mathrm{VB}$, it follows that $p$ induces an isomorphism of $\pi_{i}\left(F_{m}\left(\mathbb{R} P^{2}\right)\right)$ onto $\pi_{i}\left(F_{n}\left(\mathbb{R} P^{2}\right)\right)$.

part (2) is clear, since $F_{1}\left(\mathbb{R} P^{2}\right)=\mathbb{R} P^{2}$, and parts (3) and (4) follow from $\mathrm{VB}$.

Before proving part (5), let us recall the following general facts. For $m \geq 1$, $F_{m}\left(\mathbb{R} P^{2}\right)$ is a $2 m$-dimensional smooth manifold [CJ], and so there exists a CWcomplex $K_{m}$ (of dimension less than or equal to $2 m$ ) whose homotopy type is 
that of $F_{m}\left(\mathbb{R} P^{2}\right)$ Hi]. Let $\widetilde{K_{m}}$ (respectively $\left.\widetilde{F_{m}\left(\mathbb{R} P^{2}\right.}\right)$ ) denote the universal covering of $K_{m}$ (respectively $F_{m}\left(\mathbb{R} P^{2}\right)$ ). Then $\widetilde{K_{m}}$ is also a CW-complex whose homotopy type is that of $F_{m}\left(\mathbb{R} P^{2}\right)$ Hi].

To prove part (5), we first prove the statement for $n=2$. Let $\left\{\begin{array}{l}\mathbb{S}^{2} \longrightarrow \mathbb{R} P^{2} \\ v \longmapsto[v]\end{array}\right.$ denote the usual covering map which identifies antipodal points. Consider the $\operatorname{map}\left\{\begin{array}{c}\varphi: V_{3,2} \longrightarrow F_{2}\left(\mathbb{R} P^{2}\right) \\ \left(e_{1}, e_{2}\right) \longmapsto\left(\left[e_{1}\right],\left[e_{2}\right]\right)\end{array}\right.$ where $V_{3,2}$ is the Stiefel manifold of orthogonal 2 -frames in Euclidean 3 -space. Then $\varphi: V_{3,2} \longrightarrow \varphi\left(V_{3,2}\right)$ is a covering map with discrete fibre consisting of 4 points, and so $V_{3,2}$ and $\varphi\left(V_{3,2}\right)$ have the same homotopy type. Further, $\varphi\left(V_{3,2}\right)$ is a deformation retract of $F_{2}\left(\mathbb{R} P^{2}\right)$; indeed, if $\left(\left[v_{1}\right],\left[v_{2}\right]\right) \in F_{2}\left(\mathbb{R} P^{2}\right)$ then one may obtain an element of $\varphi\left(V_{3,2}\right)$ by deforming the second vector $v_{2}$ within the plane containing $v_{1}$ and $v_{2}$ to a vector $v_{2}^{\prime}$ orthogonal to $v_{1}$. Although $v_{2}^{\prime}$ is not unique in $V_{3,2}$, its image in $\mathbb{R} P^{2}$ is, and this gives rise to a (well-defined) deformation retract of $F_{2}\left(\mathbb{R} P^{2}\right)$ onto $\varphi\left(V_{3,2}\right)$. It follows that $V_{3,2}$ and $F_{2}\left(\mathbb{R} P^{2}\right)$ have the same homotopy type. So their universal covers do too, and the result follows from the fact that $V_{3,2}$ is homeomorphic to $\mathbb{R} P^{3}$ whose universal covering is $\mathbb{S}^{3}$.

Now suppose that $n \geq 3$. Consider the fibration $p: F_{n}\left(\mathbb{R} P^{2}\right) \longrightarrow F_{2}\left(\mathbb{R} P^{2}\right)$. By part (11), $p$ induces an isomorphism of the $i^{\text {th }}$ homotopy groups of $F_{n}\left(\mathbb{R} P^{2}\right)$ and $F_{2}\left(\mathbb{R} P^{2}\right)$ for $i \geq 2$. Composing $p$ appropriately with homotopy equivalences between $K_{n}$ and $F_{n}\left(\mathbb{R} P^{2}\right)$, and $K_{2}$ and $F_{2}\left(\mathbb{R} P^{2}\right)$, it follows that there exists a map $K_{n} \longrightarrow K_{2}$ which induces an isomorphism of their $i^{\text {th }}$ homotopy groups for $i \geq 2$, and thus a map $\widetilde{K_{n}} \longrightarrow \widetilde{K_{2}}$ which induces an isomorphism of their $i^{\text {th }}$ homotopy groups for $i \geq 2$. From Whitehead's theorem, we see that $\widetilde{K_{n}}$ and $\widetilde{K_{2}}$ are homotopy equivalent Wh2. Since $\widetilde{K_{n}}$ and $\left.\widetilde{F_{n}\left(\mathbb{R} P^{2}\right.}\right)$ (respectively $\widetilde{K_{2}}$ and $\left.\widetilde{F_{2}\left(\mathbb{R} P^{2}\right)}\right)$ have the same homotopy type, then so do $\widetilde{F_{n}\left(\mathbb{R} P^{2}\right)}$ and $\widetilde{F_{2}\left(\mathbb{R} P^{2}\right)}$, and this completes the proof of part (5).

Finally, for part (6), consider the proof of part (15) in the case $n=2$. The covering map $\varphi: V_{3,2} \longrightarrow \varphi\left(V_{3,2}\right)$ is induced by the natural free action of $\mathbb{Z}_{2} \oplus \mathbb{Z}_{2}$ on $V_{3,2}$ given as follows: if $\varepsilon_{i} \in\{ \pm 1\}$ for $i=1,2$ then $\left(\varepsilon_{1}, \varepsilon_{2}\right) \cdot\left(e_{1}, e_{2}\right)=$ $\left(\varepsilon_{1} e_{1}, \varepsilon_{2} e_{2}\right)$. Recall that $\mathbb{S}^{3}$ may be considered as the topological group of quaternions of unit modulus. One may consider the quotient of $\mathbb{S}^{3}$ arising from the standard action of $Q_{8}$ in two stages: first quotient $\mathbb{S}^{3}$ by the centre $\mathbb{Z}_{2}$ of $Q_{8}$ to obtain $\mathbb{R} P^{3}$ which is homeomorphic to $V_{3,2}$, then quotient $V_{3,2}$ by $Q_{8} / \mathbb{Z}_{2} \cong \mathbb{Z}_{2} \oplus \mathbb{Z}_{2}$. We saw above that this quotient has the same homotopy 
type as $F_{2}\left(\mathbb{R} P^{2}\right)$, and this completes the proof of part (6) and thus that of the proposition.

\subsection{Generalities on the splitting problem}

We now consider the problem of determining a cross-section to the fibration $F_{m}\left(\mathbb{R} P^{2}\right) \stackrel{p}{\longrightarrow} F_{n}\left(\mathbb{R} P^{2}\right)$ defined in Section 1 If $n \geq 2$ then this problem is equivalent to showing that the corresponding pure braid group short exact sequence splits:

Proposition 7 Let $m>n \geq 2$. Then the fibration $p: F_{m}\left(\mathbb{R} P^{2}\right) \longrightarrow F_{n}\left(\mathbb{R} P^{2}\right)$ admits a cross-section if and only if the corresponding pure braid group short exact sequence

$$
1 \longrightarrow P_{m-n}\left(\mathbb{R} P^{2} \backslash\left\{x_{1}, \ldots, x_{n}\right\}\right) \longrightarrow P_{m}\left(\mathbb{R} P^{2}\right) \stackrel{p_{*}}{\longrightarrow} P_{n}\left(\mathbb{R} P^{2}\right) \longrightarrow 1
$$

splits.

Proof The 'only if' part is clear. For the 'if part', the existence of the given short exact sequence and its splitting imply that we can find a section over the 1-skeleton, and then over the 2-skeleton of $F_{n}\left(\mathbb{R} P^{2}\right)$ [Ba, Theorem 4.3.1]. From [Wh1, the obstructions to extending the map over the $q$-skeleta, $q \geq 3$, lie in cohomology classes whose coefficients belong to the $(q-1)^{\text {th }}$ homotopy group of the fibre $F_{m-n}\left(\mathbb{R} P^{2} \backslash\left\{x_{1}, \ldots, x_{n}\right\}\right)$. But this fibre is an EilenbergMacLane space. Hence we can extend to a section over the entire space, and the result follows.

The cases not covered by Proposition 7 may be resolved as follows:

\section{Proposition 8}

(1) Let $m \geq 2$. Then the surjective group homomorphism $p_{*}: P_{m}\left(\mathbb{R} P^{2}\right) \longrightarrow$ $P_{1}\left(\mathbb{R} P^{2}\right)$ does not admit a section.

(2) Let $m \geq 2$. Then the fibration $p: F_{m}\left(\mathbb{R} P^{2}\right) \longrightarrow F_{1}\left(\mathbb{R} P^{2}\right)$ does not admit a cross-section.

Proof (1) We first note that by the commutativity of the following diagram,

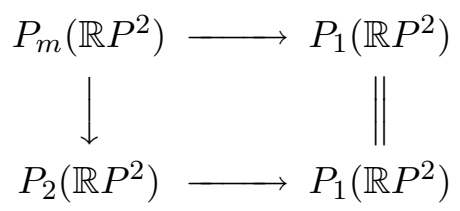


the existence of a section for $P_{m}\left(\mathbb{R} P^{2}\right) \longrightarrow P_{1}\left(\mathbb{R} P^{2}\right)$ implies that there exists a section for $P_{2}\left(\mathbb{R} P^{2}\right) \longrightarrow P_{1}\left(\mathbb{R} P^{2}\right)$ (cf [GG1, Proposition 3]), and hence it suffices to suppose that $m=2$. To see that $P_{2}\left(\mathbb{R} P^{2}\right) \longrightarrow$ $P_{1}\left(\mathbb{R} P^{2}\right)$ does not admit a section, notice that $P_{2}\left(\mathbb{R} P^{2}\right) \cong Q_{8}$ has only one element of order 2 , the full twist $\Delta_{2}$. But this element is sent to the trivial element of $P_{1}\left(\mathbb{R} P^{2}\right) \cong \mathbb{Z}_{2}$ under the projection onto $P_{1}\left(\mathbb{R} P^{2}\right)$, and so there is no section.

(2) If there were to exist a cross-section for $F_{m}\left(\mathbb{R} P^{2}\right) \longrightarrow F_{1}\left(\mathbb{R} P^{2}\right)$ then there would exist a section for $P_{m}\left(\mathbb{R} P^{2}\right) \longrightarrow P_{1}\left(\mathbb{R} P^{2}\right)$, which contradicts part (11).

Remark 9 The fact that the fibration given in part (2) of Proposition [ 8 does not admit a cross-section also follows from the fact that $\mathbb{R} P^{2}$ has the fixed point property (see also $[\mathrm{VB}]$ ).

\subsection{The splitting problem for $n \in\{2,3\}$, and the structure of $P_{3}\left(\mathbb{R} P^{2}\right)$}

This problem of the existence of a cross-section for the fibrations $F_{m}(M) \longrightarrow$ $F_{n}(M)$ was considered by Fadell and Neuwirth during the 1960's [Fa, FaN]. The case $M=\mathbb{R} P^{2}$ was later studied by Van Buskirk who constructed an explicit cross-section when $m=3$ and $n=2[\mathrm{VB}$. He posed the question of the characterisation of the values of $m$ and $n$ for which there exists a cross-section. As far as we know, if $m \geq 4$ and $n \geq 2$, the question is still completely open.

We first consider the case $m=3$ and $n=2$ of Van Buskirk, and construct an explicit splitting of the corresponding pure braid group short exact sequence. This will be useful later on when we study $P_{3}\left(\mathbb{R} P^{2}\right)$ in more detail. We show that for $m>3$ and $n=2,3$, the pure braid group short exact sequence does not split. We obtain this result by showing that for $m>3$, the fibration $F_{m}\left(\mathbb{R} P^{2}\right) \longrightarrow F_{2}\left(\mathbb{R} P^{2}\right)$ does not admit a cross-section.

The cross-section $\sigma: F_{2}\left(\mathbb{R} P^{2}\right) \longrightarrow F_{3}\left(\mathbb{R} P^{2}\right)$ defined by Van Buskirk to the fibration $F_{3}\left(\mathbb{R} P^{2}\right) \longrightarrow F_{2}\left(\mathbb{R} P^{2}\right)$ is given by $\sigma\left(\left[v_{1}\right],\left[v_{2}\right]\right)=\left(\left[v_{1}\right],\left[v_{2}\right],\left[v_{1} \wedge v_{2}\right]\right)$, where $\Lambda$ is the vector product (cf the proof of part (5) of Proposition 6). Hence the following short exact sequence splits:

$$
1 \longrightarrow \pi_{1}\left(\mathbb{R} P^{2} \backslash\left\{x_{1}, x_{2}\right\}\right) \longrightarrow P_{3}\left(\mathbb{R} P^{2}\right) \stackrel{p_{*}}{\longrightarrow} P_{2}\left(\mathbb{R} P^{2}\right) \longrightarrow 1,
$$

where $p$ is induced by the projection $F_{3}\left(\mathbb{R} P^{2}\right) \longrightarrow F_{2}\left(\mathbb{R} P^{2}\right)$ onto the last two coordinates. Since the kernel is isomorphic to $\mathbb{F}_{2}$, the free group on two 
generators, it follows that $P_{3}\left(\mathbb{R} P^{2}\right)$ is isomorphic to the semi-direct product of $\mathbb{F}_{2}$ with $Q_{8}$.

If $n \in \mathbb{N}$, a generating set for $P_{n}\left(\mathbb{R} P^{2}\right)=\pi_{1}\left(F_{n}\left(\mathbb{R} P^{2}\right),\left(x_{1}, \ldots, x_{n}\right)\right)$ may be obtained as follows. By removing a disc from $\mathbb{R} P^{2}$, one obtains a disc with a twisted band attached. For $1 \leq i \leq n$, let $\rho_{i}$ be a braid represented by the loop based at the point $x_{i}$ which goes round the band, while the other points $x_{j}, j \neq i$, stay fixed. Let $\mathbb{D}^{2} \subseteq \mathbb{R} P^{2}$ be a topological disc which contains $\left\{x_{1}, \ldots, x_{n}\right\}$. This inclusion induces a (non-injective) homomorphism $B_{n}\left(\mathbb{D}^{2}\right) \longrightarrow B_{n}\left(\mathbb{R} P^{2}\right)$. Let $\sigma_{1}, \ldots, \sigma_{n-1} \in B_{n}\left(\mathbb{R} P^{2}\right)$ be the images of the usual Artin generators of $B_{n}\left(\mathbb{D}^{2}\right)$ under this homomorphism. For $1 \leq j<k \leq n$, set $B_{j, k}=\sigma_{k-1} \cdots \sigma_{j+1} \sigma_{j}^{2} \sigma_{j+1}^{-1} \cdots \sigma_{k-1}^{-1} \in P_{n}\left(\mathbb{R} P^{2}\right)$. Then the union of the $\rho_{i}$ and $B_{j, k}$ generates $P_{n}\left(\mathbb{R} P^{2}\right)(\operatorname{cf}[\mathrm{VB},[\mathrm{S}])$.

From equation (2), we may obtain a presentation of $P_{3}\left(\mathbb{R} P^{2}\right)$, using the fact that it is an extension [J]. It has generators $\left\{\rho_{1}, \rho_{2}, \rho_{3}, B_{2,3}\right\}$ where $\left\{\rho_{1}, \rho_{2}\right\}$ are (coset representatives of) generators of the quotient $P_{2}\left(\mathbb{R} P^{2}\right)$, and $\left\{\rho_{3}, B_{2,3}\right\}$ generates the kernel. A set of relations is given by:

$$
\left.\begin{array}{rlrl}
\rho_{1} B_{2,3} \rho_{1}^{-1} & =B_{2,3} & \rho_{2} B_{2,3} \rho_{2}^{-1} & =\rho_{3}^{-1} B_{2,3}^{-1} \rho_{3} \\
\rho_{1}^{-1} B_{2,3} \rho_{1} & =B_{2,3} & \rho_{2}^{-1} B_{2,3} \rho_{2} & =B_{2,3}^{-1} \rho_{3} B_{2,3} \rho_{3}^{-1} B_{2,3} \\
\rho_{1} \rho_{3} \rho_{1}^{-1} & =\rho_{3}^{-1} B_{2,3} & \rho_{2} \rho_{3} \rho_{2}^{-1} & =\rho_{3}^{-1} B_{2,3}^{-1} \rho_{3}^{2} \\
\rho_{1}^{-1} \rho_{3} \rho_{1} & =B_{2,3} \rho_{3}^{-1} & \rho_{2}^{-1} \rho_{3} \rho_{2} & =B_{2,3}^{-1} \rho_{3} \\
\rho_{1} \rho_{2} \rho_{1} \rho_{2}^{-1} & =B_{2,3}^{-1} \rho_{3}^{2} & \rho_{1}^{-2} \rho_{2}^{2} & =B_{2,3} \rho_{3}^{-2} B_{2,3} .
\end{array}\right\}
$$

This presentation was obtained using the following useful relations:

$$
\rho_{1}^{2}=B_{1,2} B_{1,3}, \quad \rho_{2}^{2}=B_{1,2} B_{2,3}, \quad \rho_{3}^{2}=B_{1,3} B_{2,3},
$$

and the presentation $\left\langle\rho_{1}, \rho_{2} \mid \rho_{1}^{2}=\rho_{2}^{2}, \rho_{2} \rho_{1} \rho_{2}^{-1}=\rho_{1}^{-1}\right\rangle$ of $B_{2}\left(\mathbb{R} P^{2}\right)$.

Now we will define an algebraic section. It will be also be used in the following section to study properties of the torsion elements of $P_{3}\left(\mathbb{R} P^{2}\right)$.

Proposition 10 The function $s:\left\{\rho_{1}, \rho_{2}\right\} \longrightarrow P_{3}\left(\mathbb{R} P^{2}\right)$ defined by $s\left(\rho_{1}\right)=$ $\rho_{1} \rho_{3}$ and $s\left(\rho_{2}\right)=\rho_{3} \rho_{2}$ extends to a homomorphism $s: P_{2}\left(\mathbb{R} P^{2}\right) \longrightarrow P_{3}\left(\mathbb{R} P^{2}\right)$, and gives a splitting of the short exact sequence (2).

Proof The set $\left\{\rho_{1}, \rho_{2}\right\}$ generates the group $P_{2}\left(\mathbb{R} P^{2}\right)$. One may check that $s\left(\rho_{1}^{2}\right)=s\left(\rho_{2}^{2}\right)$ and $s\left(\rho_{2} \rho_{1} \rho_{2}^{-1}\right)=s\left(\rho_{1}^{-1}\right)$. Since $P_{2}\left(\mathbb{R} P^{2}\right) \cong Q_{8}$, this implies that $s$ is a homomorphism which composed with the homomorphism $p_{*}$ of the short exact sequence (2) gives the identity. 
Corollary 11 The representation $\phi: Q_{8} \longrightarrow \operatorname{Aut}\left(\mathbb{F}_{2}\right)$ induced by the section $s$ of Proposition 10 is given by $\phi\left(\rho_{1}\right)=\chi_{1}, \phi\left(\rho_{2}\right)=\chi_{2}$, and $\phi\left(\rho_{1} \rho_{2}\right)=\chi_{3}$, where:

$$
\begin{array}{ll}
\chi_{1}(x)=y & \chi_{1}(y)=x, \\
\chi_{2}(x)=y^{-1} & \chi_{2}(y)=x^{-1}, \\
\chi_{3}(x)=x^{-1} & \chi_{3}(y)=y^{-1},
\end{array}
$$

and where $x=\rho_{3}$ and $y=\rho_{3}^{-1} B_{2,3}$ form a basis of $\mathbb{F}_{2}$.

Proof From above, we know that $\left\{\rho_{3}^{-1}, B_{2,3}\right\}$ is a basis of $\mathbb{F}_{2}$. The homomorphism $s$ induces a representation $\phi$ of $Q_{8}$ in the group of automorphisms of $\mathbb{F}_{2}$. For $i=1,2$, let $\chi_{i}$ denote the automorphism $\phi\left(\rho_{i}\right)$. Then we obtain:

$\chi_{1}\left(\rho_{3}\right)=\rho_{3}^{-1} B_{2,3}, \quad \chi_{1}\left(B_{2,3}\right)=\rho_{3}^{-1} B_{2,3} \rho_{3}, \quad \chi_{2}\left(\rho_{3}\right)=B_{2,3}^{-1} \rho_{3}, \quad \chi_{2}\left(B_{2,3}\right)=B_{2,3}^{-1}$.

Consider the basis $x=\rho_{3}, y=\rho_{3}^{-1} B_{2,3}$ of $\mathbb{F}_{2}$. With respect to $\{x, y\}$, the automorphisms $\chi_{1}, \chi_{2}$ and $\chi_{3}=\chi_{2} \circ \chi_{1}$ are as given in the statement of the corollary.

As a preliminary step to proving Theorem 2 let us show that $P_{4}\left(\mathbb{R} P^{2}\right) \stackrel{p_{*}}{\longrightarrow}$ $P_{2}\left(\mathbb{R} P^{2}\right)$ does not split.

Proposition 12 Let $f_{1}, f_{2}: F_{2}\left(\mathbb{R} P^{2}\right) \longrightarrow \mathbb{R} P^{2}$ be a pair of coincidence-free maps such that the induced homomorphism $f_{1 *}: P_{2}\left(\mathbb{R} P^{2}\right) \longrightarrow P_{1}\left(\mathbb{R} P^{2}\right)$ is surjective. Then $f_{2 *}: P_{2}\left(\mathbb{R} P^{2}\right) \longrightarrow P_{1}\left(\mathbb{R} P^{2}\right)$ is also surjective, and $f_{1 *} \neq f_{2 *}$ ie, the induced homomorphisms on the fundamental group are different and both non trivial.

Proof Consider the map $\left\{\begin{aligned}\left(f_{1}, f_{2}\right): F_{2}\left(\mathbb{R} P^{2}\right) & \longrightarrow \mathbb{R} P^{2} \times \mathbb{R} P^{2} \\ w & \longmapsto\left(f_{1}(w), f_{2}(w)\right) .\end{aligned}\right.$ If $\left(f_{1}, f_{2}\right)$ is coincidence free (or can be deformed to a coincidence-free pair) then there is a factorisation via $F_{2}\left(\mathbb{R} P^{2}\right)=\mathbb{R} P^{2} \times \mathbb{R} P^{2} \backslash \Delta$, where $\Delta$ is the diagonal, and so there exists a homomorphism $\phi: P_{2}\left(\mathbb{R} P^{2}\right) \longrightarrow P_{2}\left(\mathbb{R} P^{2}\right)$ which makes the following diagram commute:

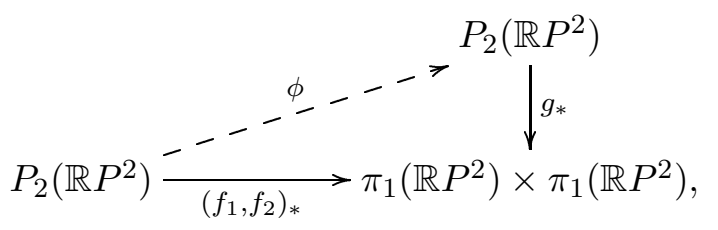


where $g_{*}$ is the homomorphism induced by the inclusion $g: F_{2}\left(\mathbb{R} P^{2}\right) \longleftrightarrow$ $\mathbb{R} P^{2} \times \mathbb{R} P^{2}$. Identifying $\pi_{1}\left(\mathbb{R} P^{2} \times \mathbb{R} P^{2}\right)$ with $\mathbb{Z}_{2}+\mathbb{Z}_{2}$ in the obvious way, let $\rho_{1}, \rho_{2}$ be generators of $P_{2}\left(\mathbb{R} P^{2}\right)$ such that $g_{*}\left(\rho_{1}\right)=(1,0)$ and $g_{*}\left(\rho_{2}\right)=(0,1)$. Let us first show that $f_{2 *}$ cannot be the trivial homomorphism. Suppose on the contrary that $f_{2 *}=0$. If $a \in P_{2}\left(\mathbb{R} P^{2}\right)$ then there exist $i \in\{0,1,2,3\}$ and $j \in\{0,1\}$ such that $\phi(a)=\rho_{1}^{i} \rho_{2}^{j}$. Since $g_{*} \circ \phi=\left(f_{1}, f_{2}\right)_{*}$, it follows that $j=0$. Now $f_{1 *}$ is surjective, so there exist $b \in\left\{\rho_{1}, \rho_{2}\right\}$ and $\varepsilon \in\{ \pm 1\}$ such that $\phi(b)=\rho_{1}^{\varepsilon}$. Hence $P_{2}\left(\mathbb{R} P^{2}\right) / \operatorname{Ker}(\phi) \cong \mathbb{Z}_{4}$. But $P_{2}\left(\mathbb{R} P^{2}\right) \cong Q_{8}$ of which the only quotient of order 4 is $\mathbb{Z}_{2}+\mathbb{Z}_{2}$ - a contradiction. Since $P_{1}\left(\mathbb{R} P^{2}\right) \cong \mathbb{Z}_{2}$, we conclude that $f_{2 *}$ is surjective.

Suppose now that $f_{1 *}=f_{2 *}$. Let $a \in P_{2}\left(\mathbb{R} P^{2}\right)$. Then there exist $i \in\{0,1,2,3\}$ and $j \in\{0,1\}$ such that $\phi(a)=\rho_{1}^{i} \rho_{2}^{j}$. Since $g_{*} \circ \phi=\left(f_{1}, f_{2}\right)_{*}$, it follows that $i \equiv j \bmod 2$. Now $f_{1 *}$ is surjective, so there exist $b \in\left\{\rho_{1}, \rho_{2}\right\}$ and $\varepsilon \in\{ \pm 1\}$ such that $\phi(b)=\rho_{1}^{\varepsilon} \rho_{2}$. By an argument similar to that of the previous paragraph, we obtain a contradiction, whence $f_{1 *} \neq f_{2 *}$.

We are now able to prove the main results of this section.

Proof of Theorem 2(1) As in the proof of part (2) of Proposition 8, it suffices to show that there is no section for $m=4$. Suppose on the contrary that such a section exists. Then there exists a map $\xi: F_{2}\left(\mathbb{R} P^{2}\right) \longrightarrow$ $F_{4}\left(\mathbb{R} P^{2}\right)$ of the form $\xi\left(x_{1}, x_{2}\right)=\left(x_{1}, x_{2}, f_{1}\left(x_{1}, x_{2}\right), f_{2}\left(x_{1}, x_{2}\right)\right)$. For $i=1,2$, let $p_{i}: F_{2}\left(\mathbb{R} P^{2}\right) \longrightarrow \mathbb{R} P^{2}$ denote the map $p_{i}\left(x_{1}, x_{2}\right)=x_{i}$. Then the four maps $f_{1}, f_{2}, p_{1}, p_{2}: F_{2}\left(\mathbb{R} P^{2}\right) \longrightarrow \mathbb{R} P^{2}$ are pairwise coincidence free, and so by Proposition [12, the induced homomorphisms are all surjective and pairwise distinct. But, since $P_{2}\left(\mathbb{R} P^{2}\right) \cong Q_{8}$ and $P_{1}\left(\mathbb{R} P^{2}\right) \cong \mathbb{Z}_{2}$, it follows that there are only three surjective homomorphisms $P_{2}\left(\mathbb{R} P^{2}\right) \longrightarrow P_{1}\left(\mathbb{R} P^{2}\right)$. We thus obtain a contradiction, and the result follows.

Proof of Theorem 3 First let $n=2$. By Proposition [7 if this sequence were to split then there would exist a cross-section to the given fibration. But this would contradict Theorem 2(1).

Now let $n=3$. Consider the following commutative diagram:

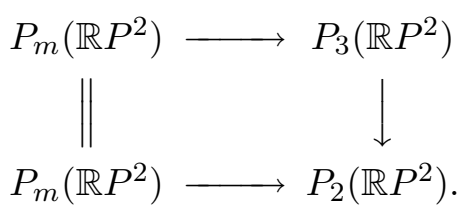


Since the right-hand vertical arrow admits a section, if there were to exist a section for $P_{m}\left(\mathbb{R} P^{2}\right) \longrightarrow P_{3}\left(\mathbb{R} P^{2}\right)$ then there would also exist a section for $P_{m}\left(\mathbb{R} P^{2}\right) \longrightarrow P_{2}\left(\mathbb{R} P^{2}\right)$ which contradicts the previous paragraph.

Proof of Theorem 2(2) The result follows from Proposition 7 and Theorem 3. This also completes the proof of Theorem 2.

\section{Torsion elements and roots of the full twist in $P_{n}\left(\mathbb{R} P^{2}\right)$}

Let $\Delta_{n}$ denote the full twist braid of $B_{n}\left(\mathbb{R} P^{2}\right)$ as defined in equation (11). In $[\mathrm{M}]$, Murasugi gave a characterisation of the torsion elements of $B_{n}\left(\mathbb{R} P^{2}\right)$ and the quotient of $B_{n}\left(\mathbb{R} P^{2}\right)$ by its centre $\left\langle\Delta_{n}\right\rangle$. However, the orders of the elements are not clear, even in the case of $P_{n}\left(\mathbb{R} P^{2}\right)$. In this section, we study the torsion and the roots of $\Delta_{n}$ in $P_{n}\left(\mathbb{R} P^{2}\right)$ with methods different to those of Murasugi. Using the pure braid group short exact sequence, we first show that the possible torsion of $P_{n}\left(\mathbb{R} P^{2}\right)$ is 2 or 4 . We then go on to prove that $\Delta_{n}$ is the unique element of $P_{n}\left(\mathbb{R} P^{2}\right)$ of order 2 , and that for all $n \geq 2, P_{n}\left(\mathbb{R} P^{2}\right)$ possesses elements of order 4 . It will follow that the full twist possesses $k^{\text {th }}$ roots in $P_{n}\left(\mathbb{R} P^{2}\right), k \geq 2$, if and only if $k=2$. Finally, using the semi-direct product decomposition given in Proposition 10, we study in more detail the torsion elements of $P_{3}\left(\mathbb{R} P^{2}\right)$.

We first observe that the possible torsion of $P_{n}\left(\mathbb{R} P^{2}\right)$ is either 2 or 4 .

Lemma 13 Let $1 \longrightarrow K \longrightarrow G \stackrel{f}{\longrightarrow} H \longrightarrow 1$ be a short exact sequence of groups such that $K$ is torsion free. If $x \in G$ is a torsion element of order $l$, then the element $f(x)$ of $H$ has also order $l$.

Proof Let $x \in G$ be a torsion element of order $l$. Therefore $f(x)^{l}=1$, and the order $k$ of $f(x)$ divides $l$. Consider $x^{k}$. It belongs to $\operatorname{Ker}(f)=K$, and $\left(x^{k}\right)^{\frac{l}{k}}=1$. Since $K$ is torsion free, it follows that $x^{k}=1$, and we conclude that $k=l$.

Corollary 14 Let $n \in \mathbb{N}$. Then the possible (non-trivial) torsion of $P_{n}\left(\mathbb{R} P^{2}\right)$ is 2 or 4 . 
Proof Since $P_{1}\left(\mathbb{R} P^{2}\right) \cong \mathbb{Z}_{2}$ and $P_{2}\left(\mathbb{R} P^{2}\right) \cong Q_{8}$, the result follows if $n \in$ $\{1,2\}$. If $n \geq 3$, consider the following pure braid group short exact sequence:

$$
1 \longrightarrow P_{n-2}\left(\mathbb{R} P^{2} \backslash\left\{x_{1}, x_{2}\right\}\right) \longrightarrow P_{n}\left(\mathbb{R} P^{2}\right) \stackrel{p_{*}}{\longrightarrow} P_{2}\left(\mathbb{R} P^{2}\right) \longrightarrow 1 .
$$

Now $F_{n-2}\left(\mathbb{R} P^{2} \backslash\left\{x_{1}, x_{2}\right\}\right)$ is a finite-dimensional Eilenberg-MacLane space, and so by a theorem of P. Smith, $P_{n-2}\left(\mathbb{R} P^{2} \backslash\left\{x_{1}, x_{2}\right\}\right)$ is torsion free [FaN]. The result follows from Lemma 13.

Proposition 15 Let $n \geq 2$. Then the full twist $\Delta_{n}$ is the unique element of $P_{n}\left(\mathbb{R} P^{2}\right)$ of order 2 in $P_{n}\left(\mathbb{R} P^{2}\right)$. In particular, the square of any element of $P_{n}\left(\mathbb{R} P^{2}\right)$ of order 4 is the full twist.

Proof The proof of the first part is by induction. The result is true for $n=2$, since $P_{2}\left(\mathbb{R} P^{2}\right) \cong Q_{8}$, and the unique element of order 2 of this group is the full twist. Suppose that the result is true for some $n \geq 2$. Consider the following short exact sequence:

$$
1 \longrightarrow F \longrightarrow P_{n+1}\left(\mathbb{R} P^{2}\right) \stackrel{p_{*}}{\longrightarrow} P_{n}\left(\mathbb{R} P^{2}\right) \longrightarrow 1,
$$

where $F=\pi_{1}\left(\mathbb{R} P^{2} \backslash\left\{x_{1}, \ldots, x_{n}\right\}\right)$ is torsion free. If $\alpha \in P_{n+1}\left(\mathbb{R} P^{2}\right)$ is of order 2 then $p_{*}(\alpha) \in P_{n}\left(\mathbb{R} P^{2}\right)$ is also of order 2 by Lemma 13, and so $p_{*}(\alpha)=\Delta_{n}$ by the induction hypothesis. Since $p_{*}\left(\Delta_{n+1}\right)=\Delta_{n}$, it follows that $\alpha=\Delta_{n+1}$. $w$, where $w \in F$. Then $1=\alpha^{2}=\Delta_{n+1}^{2} \cdot w^{2}=w^{2}$ since $\Delta_{n+1}$ generates $Z\left(P_{n+1}\left(\mathbb{R} P^{2}\right)\right)$ and is of order $2[\mathrm{VB},[\mathrm{M}]$. But $F$ is torsion free, thus $w=1$, and so $\Delta_{n+1}$ is the unique element of $P_{n+1}\left(\mathbb{R} P^{2}\right)$ of order 2 . The fact that the square of any element of $P_{n}\left(\mathbb{R} P^{2}\right)$ of order 4 is the full twist then follows easily.

Since $P_{2}\left(\mathbb{R} P^{2}\right)$ has an element of order 4 , it follows from Proposition [10 that $P_{3}\left(\mathbb{R} P^{2}\right)$ does too. In spite of the fact that $P_{n}\left(\mathbb{R} P^{2}\right) \stackrel{p_{*}}{\longrightarrow} P_{2}\left(\mathbb{R} P^{2}\right)$ does not admit a section, we will show that $P_{n}\left(\mathbb{R} P^{2}\right)$ contains elements of order 4 for all $n \geq 2$. We first recall a presentation of $B_{n}\left(\mathbb{R} P^{2}\right)$.

Proposition 16 (Van Buskirk $[\mathrm{VB}$ ) The following constitutes a presentation of the group $B_{n}\left(\mathbb{R} P^{2}\right)$ :

\section{Generators}

$$
\sigma_{1}, \ldots, \sigma_{n-1}, \rho_{1}, \ldots, \rho_{n} .
$$




\section{Relations}

$$
\begin{aligned}
\sigma_{i} \sigma_{j} & =\sigma_{j} \sigma_{i} \quad \text { if }|i-j| \geq 2, \\
\sigma_{i} \sigma_{i+1} \sigma_{i} & =\sigma_{i+1} \sigma_{i} \sigma_{i+1} \quad \text { for } 1 \leq i \leq n-2, \\
\sigma_{i} \rho_{j} & =\rho_{j} \sigma_{i} \quad \text { for } j \neq i, i+1, \\
\rho_{i+1} & =\sigma_{i}^{-1} \rho_{i} \sigma_{i}^{-1} \quad \text { for } 1 \leq i \leq n-1, \\
\rho_{i+1}^{-1} \rho_{i}^{-1} \rho_{i+1} \rho_{i} & =\sigma_{i}^{2} \quad \text { for } 1 \leq i \leq n-1, \\
\rho_{1}^{2} & =\sigma_{1} \sigma_{2} \cdots \sigma_{n-2} \sigma_{n-1}^{2} \sigma_{n-2} \cdots \sigma_{2} \sigma_{1} .
\end{aligned}
$$

We warn the reader that the $\rho_{i}$ of Proposition 16 are slightly different from the $\rho_{i}$ defined in Section 2.3. From this presentation, if $2 \leq j \leq n$, we obtain the relation

$$
\rho_{j}=\sigma_{j-1}^{-1} \cdots \sigma_{1}^{-1} \rho_{1} \sigma_{1}^{-1} \cdots \sigma_{j-1}^{-1} .
$$

Notice also that $B_{i, j+1}=\sigma_{j} B_{i, j} \sigma_{j}^{-1}$ for all $1 \leq i<j \leq n-1$.

Lemma 17 Let $1 \leq i<j \leq n$. Then $\rho_{j} \rho_{i}=\rho_{i} \rho_{j} B_{i, j}$.

Proof Let $1 \leq i \leq n-1$. The proof is by induction on $j-i$. Suppose first that $j-i=1$. Then $\rho_{i+1} \rho_{i}=\rho_{i} \rho_{i+1} \sigma_{i}^{2}=\rho_{i} \rho_{i+1} B_{i, i+1}$ by Proposition 16. Suppose now that the given equation holds for $i$ and $j$ satisfying $1 \leq i<j \leq n-1$ and $1 \leq j-i \leq n-2$. Applying Proposition [16, we have:

$$
\begin{aligned}
\rho_{j+1} \rho_{i} & =\sigma_{j}^{-1} \rho_{j} \sigma_{j}^{-1} \rho_{i}=\sigma_{j}^{-1} \rho_{j} \rho_{i} \sigma_{j}^{-1}=\sigma_{j}^{-1} \rho_{i} \rho_{j} B_{i, j} \sigma_{j}^{-1} \\
& =\rho_{i} \sigma_{j}^{-1} \rho_{j} \sigma_{j}^{-1} \sigma_{j} B_{i, j} \sigma_{j}^{-1}=\rho_{i} \rho_{j+1} B_{i, j+1},
\end{aligned}
$$

which proves the lemma.

Proposition 18 Let $n \in \mathbb{N}$. In $B_{n}\left(\mathbb{R} P^{2}\right),\left(\rho_{n-1} \cdots \rho_{1}\right)^{2}=\Delta_{n}$.

Proof If $n=1$ then both sides are trivial, and the result is true. So let $n \geq 2$. Let us first show that $\left(\rho_{n-1} \cdots \rho_{1}\right)^{2}$ is central in $B_{n}\left(\mathbb{R} P^{2}\right)$. From Proposition [16] it suffices to prove that it commutes with each member of the generating set $\left\{\sigma_{1}, \ldots, \sigma_{n-1}, \rho_{n}\right\}$. By Lemma 17 we have that $\sigma_{i} \rho_{i+1} \rho_{i} \sigma_{i}^{-1}=$ $\sigma_{i} \rho_{i} \rho_{i+1} \sigma_{i}=\sigma_{i}^{2} \cdot \sigma_{i}^{-1} \rho_{i} \sigma_{i}^{-1} \cdot \sigma_{i} \rho_{i+1} \sigma_{i}=\sigma_{i}^{2} \rho_{i+1} \rho_{i}$. Applying Proposition 16] we obtain:

$$
\sigma_{i}\left(\rho_{n-1} \cdots \rho_{1}\right) \sigma_{i}^{-1}=\rho_{n-1} \cdots \rho_{i+2} \sigma_{i} \rho_{i+1} \rho_{i} \sigma_{i}^{-1} \rho_{i-1} \cdots \rho_{1}=\sigma_{i}^{2}\left(\rho_{n-1} \cdots \rho_{1}\right) .
$$

Hence:

$$
\sigma_{i}\left(\rho_{n-1} \cdots \rho_{1}\right)=\left(\rho_{n-1} \cdots \rho_{1}\right) \sigma_{i}^{-1}
$$


and so $\sigma_{i}$ commutes with $\left(\rho_{n-1} \cdots \rho_{1}\right)^{2}$. Now let us show that $\rho_{n}$ commutes with $\left(\rho_{n-1} \cdots \rho_{1}\right)^{2}$. Set $w=B_{1, n} \cdots B_{n-2, n} B_{n-1, n}$. Using Lemma 17 and Proposition [16, we see that

$$
\begin{aligned}
\left(\rho_{1}^{-1} \cdots \rho_{n-1}^{-1}\right) \rho_{n}\left(\rho_{n-1} \cdots \rho_{1}\right) & =\left(\rho_{1}^{-1} \cdots \rho_{n-2}^{-1}\right) \rho_{n}\left(\rho_{n-2} \cdots \rho_{1}\right) B_{n-1, n} \\
& =\left(\rho_{1}^{-1} \cdots \rho_{n-3}^{-1}\right) \rho_{n}\left(\rho_{n-3} \cdots \rho_{1}\right) B_{n-2, n} B_{n-1, n} \\
& =\rho_{n} w .
\end{aligned}
$$

The relation $w=\sigma_{n-1} \cdots \sigma_{2} \sigma_{1}^{2} \sigma_{2} \cdots \sigma_{n-1}$ holds in $B_{n}\left(\mathbb{R} P^{2}\right)$ since it holds in $B_{n}\left(\mathbb{D}^{2}\right)$. Thus $\left(\rho_{1}^{-1} \cdots \rho_{n-1}^{-1}\right) w\left(\rho_{n-1} \cdots \rho_{1}\right)=w^{-1}$ using equation (15), and so from equation (6), we obtain

$$
\begin{aligned}
\left(\rho_{1}^{-1} \cdots \rho_{n-1}^{-1}\right)^{2} \rho_{n}\left(\rho_{n-1} \cdots \rho_{1}\right)^{2} & =\left(\rho_{1}^{-1} \cdots \rho_{n-1}^{-1}\right) \rho_{n} w\left(\rho_{n-1} \cdots \rho_{1}\right) \\
& =\rho_{n} w w^{-1}=\rho_{n},
\end{aligned}
$$

which shows that $\rho_{n}$ commutes with $\left(\rho_{n-1} \cdots \rho_{1}\right)^{2}$.

Hence $\left(\rho_{n-1} \cdots \rho_{1}\right)^{2}$ belongs to $Z\left(B_{n}\left(\mathbb{R} P^{2}\right)\right)$, which is the cyclic subgroup of order 2 generated by $\Delta_{n}$. Suppose that $\left(\rho_{n-1} \cdots \rho_{1}\right)^{2}=1$. Clearly, $\rho_{n-1} \cdots \rho_{1} \neq$ 1 (since under the projection $P_{n}\left(\mathbb{R} P^{2}\right) \longrightarrow P_{1}\left(\mathbb{R} P^{2}\right), \rho_{n-1} \cdots \rho_{1}$ is sent to $\left.\rho_{1}\right)$. So $\rho_{n-1} \cdots \rho_{1}$ is an element of $P_{n}\left(\mathbb{R} P^{2}\right)$ of order 2. By Proposition 15 it follows that $\rho_{n-1} \cdots \rho_{1}=\Delta_{n}$. But this cannot be so, because $\Delta_{n}$ is central in $B_{n}\left(\mathbb{R} P^{2}\right)$, while by equation (5), $\rho_{n-1} \cdots \rho_{1}$ is not. We conclude that $\left(\rho_{n-1} \cdots \rho_{1}\right)^{2}=\Delta_{n}$.

Since by Proposition 18 the element $\rho_{n-1} \cdots \rho_{1}$ is of order 4 , the characterisation of the torsion of $P_{n}\left(\mathbb{R} P^{2}\right)$ thus follows from Corollary 14]

Corollary 19 Let $n \geq 2$. Then the (non-trivial) torsion of $P_{n}\left(\mathbb{R} P^{2}\right)$ is precisely 2 and 4.

We have a result analogous to that of Proposition 18 for $\rho_{n} \cdots \rho_{1}$ :

Proposition 20 Let $n \in \mathbb{N}$. In $B_{n}\left(\mathbb{R} P^{2}\right),\left(\rho_{n} \cdots \rho_{1}\right)^{2}=\Delta_{n}$.

Proof If $n=1$ then $\rho_{1}^{2}=\Delta_{1}=1$. So suppose that $n \geq 2$. First, using Proposition [16] and the definition of $w$ given in the proof of Proposition [18, we see that

$$
\begin{aligned}
\rho_{n}^{2} & =\sigma_{n-1}^{-1} \cdots \sigma_{1}^{-1} \rho_{1} \sigma_{1}^{-1} \cdots \sigma_{n-1}^{-1} \cdot \sigma_{n-1}^{-1} \cdots \sigma_{1}^{-1} \rho_{1} \sigma_{1}^{-1} \cdots \sigma_{n-1}^{-1} \\
& =\sigma_{n-1}^{-1} \cdots \sigma_{1}^{-2} \cdots \sigma_{n-1}^{-1}=w^{-1} .
\end{aligned}
$$


By Proposition [16 and Lemma 17, we observe that:

$$
\begin{aligned}
\left(\rho_{n} \cdots \rho_{1}\right)^{2} & =\rho_{n} \rho_{n-1} \cdots \rho_{1} \rho_{n-1} \rho_{n} \rho_{n-2} \cdots \rho_{1} B_{n-1, n} \\
& =\rho_{n}\left(\rho_{n-1} \cdots \rho_{1}\right)^{2} \rho_{n} w=\rho_{n} \Delta_{n} \rho_{n} w=\Delta_{n} \rho_{n}^{2} w=\Delta_{n},
\end{aligned}
$$

using equation (77) and the fact that $\Delta_{n}$ is central. The result follows.

Now we go on to study $P_{3}\left(\mathbb{R} P^{2}\right)$ in more detail, using the fact that it is a semi-direct product. Since $P_{2}\left(\mathbb{R} P^{2}\right) \cong Q_{8}, P_{2}\left(\mathbb{R} P^{2}\right)$ has 6 elements of order 4 which make up three distinct conjugacy classes. We will show that the same happens in $P_{3}\left(\mathbb{R} P^{2}\right)$.

Proposition 21 Let $p_{*}: P_{3}\left(\mathbb{R} P^{2}\right) \longrightarrow P_{2}\left(\mathbb{R} P^{2}\right)$ be the homomorphism induced by the projection onto the first two coordinates. Then:

(1) $p_{*}$ sends the set of elements of $P_{3}\left(\mathbb{R} P^{2}\right)$ of order 4 surjectively onto the set of elements of $P_{2}\left(\mathbb{R} P^{2}\right)$ of order 4 ;

(2) any two elements of $P_{3}\left(\mathbb{R} P^{2}\right)$ of order 4 which project onto the same element of $P_{2}\left(\mathbb{R} P^{2}\right)$ are conjugate;

(3) the set of elements of $P_{3}\left(\mathbb{R} P^{2}\right)$ of order 4 form three distinct conjugacy classes;

(4) if $H$ is a subgroup of $P_{3}\left(\mathbb{R} P^{2}\right)$ abstractly isomorphic to $Q_{8}$, then $p_{*}(H) \cong$ $P_{2}\left(\mathbb{R} P^{2}\right)$. Further, any two such subgroups are conjugate.

Proof The proof of this proposition will rely on the results of Section 2 notably the semi-direct product $P_{3}\left(\mathbb{R} P^{2}\right) \cong \mathbb{F}_{2} \rtimes P_{2}\left(\mathbb{R} P^{2}\right)$ given by Proposition 10 and the corresponding representation $\phi: P_{2}\left(\mathbb{R} P^{2}\right) \longrightarrow \operatorname{Aut}\left(\mathbb{F}_{2}\right)$ of Corollary [1]. If $z \in P_{2}\left(\mathbb{R} P^{2}\right)$, let us write $\phi(z)=\phi_{z}$. In what follows we shall identify $P_{3}\left(\mathbb{R} P^{2}\right)$ with $\mathbb{F}_{2} \rtimes P_{2}\left(\mathbb{R} P^{2}\right)$, in particular, if $(w, t) \in \mathbb{F}_{2} \rtimes P_{2}\left(\mathbb{R} P^{2}\right)$ then $p_{*}((w, t))=t \in P_{2}\left(\mathbb{R} P^{2}\right)$.

part (11) follows directly from the existence of the splitting. For part (2), suppose that the element $(w, t) \in \mathbb{F}_{2} \rtimes P_{2}\left(\mathbb{R} P^{2}\right)$ is of order 4 . By part (1), $t \in P_{2}\left(\mathbb{R} P^{2}\right)$ is of order 4 , and hence so is $(1, t) \in \mathbb{F}_{2} \rtimes P_{2}\left(\mathbb{R} P^{2}\right)$. To prove the result, it suffices to show that $(w, t)$ and $(1, t)$ are conjugate. From the explicit description of $\phi$ given in Corollary [1] one sees that $\phi_{t^{2}}=\operatorname{Id}_{\mathbb{F}_{2}}$, and so:

$$
(1,1)=(w, t)^{4}=\left(w \phi_{t}(w), t^{2}\right)^{2}=\left(w \phi_{t}(w) \phi_{t^{2}}\left(w \phi_{t}(w)\right), t^{4}\right)=\left(\left(w \phi_{t}(w)\right)^{2}, 1\right) .
$$

Since $\mathbb{F}_{2}$ is torsion free and $\left(w \phi_{t}(w)\right)^{2}=1$, it follows that $w \phi_{t}(w)=1$, and so $\phi_{t}(w)=w^{-1}$. Let us characterise such $w \in \mathbb{F}_{2}$. Since $\phi_{t^{2}}=\operatorname{Id}_{\mathbb{F}_{2}}$ for all $t \in P_{2}\left(\mathbb{R} P^{2}\right)$, we see that $\phi_{t^{3}}=\phi_{t}$, and so it suffices to suppose that 
$t \in\left\{\rho_{1}, \rho_{2}, \rho_{1} \rho_{2}\right\}$. Using the description of the associated automorphisms $\phi_{t}$ given in Corollary [11] we see in each of the three possible cases that $w$ is of the (reduced) form $w=w_{1} w_{2}$, where $w_{2}=\phi_{t}\left(w_{1}^{-1}\right)$. This implies that:

$\left(w_{1}, 1\right) \cdot(1, t) \cdot\left(w_{1}, 1\right)^{-1}=\left(w_{1}, 1\right) \cdot(1, t) \cdot\left(w_{1}^{-1}, 1\right)=\left(w_{1}, 1\right) \cdot\left(\phi_{t}\left(w_{1}^{-1}\right), t\right)=(w, t)$,

and part (2) follows. One may then check easily that if $(1, t)$ and $\left(1, t^{\prime}\right)$ are conjugate in $\mathbb{F}_{2} \rtimes P_{2}\left(\mathbb{R} P^{2}\right)$ then $t$ and $t^{\prime}$ are conjugate in $P_{2}\left(\mathbb{R} P^{2}\right)$. Since the set of elements of $P_{2}\left(\mathbb{R} P^{2}\right)$ of order 4 splits into three conjugacy classes, part (3) follows from parts (11) and (2).

For the first part of (4), since $H$ is a torsion subgroup and the kernel of $p_{*}$ is torsion free, it follows that $\left.p_{*}\right|_{H}: H \longrightarrow P_{2}\left(\mathbb{R} P^{2}\right)$ is injective, and thus an isomorphism. For the second part, let $K$ be the subgroup of $P_{3}\left(\mathbb{R} P^{2}\right)$ generated by $s\left(\rho_{1}\right)$ and $s\left(\rho_{2}\right)$, where $s: P_{2}\left(\mathbb{R} P^{2}\right) \longrightarrow P_{3}\left(\mathbb{R} P^{2}\right)$ is the section of Proposition 10, and let $H$ be a subgroup of $P_{3}\left(\mathbb{R} P^{2}\right)$ which is isomorphic to $Q_{8}$. To prove the statement, it suffices to prove that $H$ is conjugate to $K$. From above, $p_{*}(H)=P_{2}\left(\mathbb{R} P^{2}\right)$, so let $b \in H$ be such that $p_{*}(b)=\rho_{1}$. By part (2), there exists $\gamma \in P_{3}\left(\mathbb{R} P^{2}\right)$ such that $\gamma b \gamma^{-1}=s\left(\rho_{1}\right)$. Let $H^{\prime}=\gamma H \gamma^{-1}$. Then it suffices to prove that $H^{\prime}=K$. To do so, first notice that $s\left(\rho_{1}\right) \in H^{\prime} \cap K$. Let $c \in H^{\prime}$ be such that $p_{*}(c)=\rho_{2}$. Again by part (2), since $p_{*}\left(s\left(\rho_{2}\right)\right)=\rho_{2}$, there exists $(w, t) \in \mathbb{F}_{2} \rtimes P_{2}\left(\mathbb{R} P^{2}\right)$ such that:

$$
c=(w, t) \cdot\left(1, \rho_{2}\right) \cdot(w, t)^{-1}=\left(w \phi_{t \rho_{2} t^{-1}}\left(w^{-1}\right), t \rho_{2} t^{-1}\right) .
$$

We have once more identified $P_{3}\left(\mathbb{R} P^{2}\right)$ with $\mathbb{F}_{2} \rtimes P_{2}\left(\mathbb{R} P^{2}\right)$, in particular, $s\left(\rho_{i}\right)=$ $\left(1, \rho_{i}\right)$ for $i=1,2$. By Corollary 11, we obtain $\phi_{\rho_{2}}=\phi_{\rho_{2}^{-1}}$, and since $t \rho_{2} t^{-1}$ is of the form $\rho_{2}^{ \pm 1}$, it follows that $\phi_{t \rho_{2} t^{-1}}=\phi_{\rho_{2}}$. From equation (8), we obtain:

$$
c^{-1}=\left(w \phi_{\rho_{2}}\left(w^{-1}\right), \rho_{2}^{\mp 1}\right) .
$$

On the other hand, $s\left(\rho_{1}\right) \cdot c \cdot\left(s\left(\rho_{1}\right)\right)^{-1}=c^{-1}$ in $H^{\prime}$, so:

$$
c^{-1}=\left(1, \rho_{1}\right) \cdot\left(w \phi_{\rho_{2}}\left(w^{-1}\right), \rho_{2}^{ \pm 1}\right) \cdot\left(1, \rho_{1}\right)^{-1}=\left(\phi_{\rho_{1}}\left(w \phi_{\rho_{2}}\left(w^{-1}\right)\right), \rho_{2}^{\mp 1}\right) .
$$

Combining equations (9) and (10), we see that $w \phi_{\rho_{2}}\left(w^{-1}\right)$ is a fixed element of $\phi_{\rho_{1}}$, but by Corollary [1], the only such element is the identity. So $\phi_{\rho_{2}}\left(w^{-1}\right)=$ $w^{-1}$, and similarly we conclude that $w=1$. By equation (8), we see that $c=\left(1, \rho_{2}^{ \pm 1}\right)=s\left(\rho_{2}^{ \pm 1}\right)$, and since $H^{\prime}$ is generated by $\left\{c, s\left(\rho_{1}\right)\right\}$, it follows that $H^{\prime}=K$ as required.

Remark 22 For the case $P_{3}\left(\mathbb{R} P^{2}\right)$, our results are compatible with those of Murasugi $\left[\mathrm{M}\right.$. His classification shows that the torsion elements of $B_{3}\left(\mathbb{R} P^{2}\right)$ are, up to powers and conjugacy, $\sigma_{1} \sigma_{2}$ (order 6 ), $\sigma_{1} \sigma_{2} \sigma_{1}$ and $\rho_{1} \sigma_{2}$ (both of 
order 4), $\rho_{2} \sigma_{1}$ (order 8), and $\rho_{3} \sigma_{2} \sigma_{1}$ (order 12). The torsion elements of $P_{3}\left(\mathbb{R} P^{2}\right)$ are, up to powers and conjugacy, $\Delta_{3}$ (order 2), $\rho_{2} \rho_{1}$ and $\rho_{3} \rho_{2} \rho_{1}$ (both of order 4$)$. Then $\sigma_{2}\left(\rho_{2} \rho_{1}\right)^{ \pm 1} \sigma_{2}^{-1}=\left(\rho_{3}^{-1} \rho_{2}^{-1} \rho_{3} \rho_{2} \rho_{3} \rho_{1}\right)^{ \pm 1}$ projects onto $\rho_{1}^{ \pm 1}$, $\left(\rho_{3} \rho_{2} \rho_{1}\right)^{ \pm 1}$ projects onto $\left(\rho_{2} \rho_{1}\right)^{ \pm 1}$, and the element $\sigma_{1} \sigma_{2}\left(\rho_{2} \rho_{1}\right)^{ \pm 1} \sigma_{2}^{-1} \sigma_{1}^{-1}=$ $\left(\rho_{1}^{2} \rho_{1}^{-1} \rho_{2}^{-1} \rho_{1} \rho_{2} \rho_{3} \rho_{2}^{-1} \rho_{1}^{-1} \rho_{2} \rho_{1} \rho_{2}\right)^{ \pm 1}$ projects onto $\rho_{2}^{\mp 1}$.

\section{Roots of the full twist and torsion elements in $B_{n}\left(\mathbb{R} P^{2}\right)$}

In this section, we study the torsion of the group $B_{n}\left(\mathbb{R} P^{2}\right)$ for $n \geq 2$. In $[\mathrm{M}$, Murasugi introduces elements $A_{1}(n, r, s, q)$ and $A_{2}(n, r, s, q)$ of $B_{n}\left(\mathbb{R} P^{2}\right)$, and shows that if $r>0$ then there are the relations $m p=2 r k$ and $m q=s k$ for integers $m \neq 0$, and for these integers, we have $A_{1}(n, r, s, q)^{2 r m}=\Delta_{n}$ and $A_{2}(n, r, s, q)^{4 r m}=1$. But the order of such elements is not given. We show that the full twist $\Delta_{n}$ is the unique element of order two in $B_{n}\left(\mathbb{R} P^{2}\right)$, and then prove Theorem 4 which says that $B_{n}\left(\mathbb{R} P^{2}\right)$ has an element of order $k$ if and only if $k$ divides either $4 n$ or $4(n-1)$. As a consequence, we obtain Theorem [5 so that the full twist $\Delta_{n}$ has a $k^{\text {th }}$ root if and only if $k$ either divides $2 n$ or $2(n-1)$. Our techniques are different to those of Murasugi, in particular, we use the notion of intermediate coverings between $F_{n}\left(\mathbb{R} P^{2}\right)$ and $D_{n}\left(\mathbb{R} P^{2}\right)$ introduced in GG2.

Proposition 23 If $n \geq 2$ then the full twist $\Delta_{n}$ is the unique element of $B_{n}\left(\mathbb{R} P^{2}\right)$ of order 2 .

Proof Let $\pi: B_{n}\left(\mathbb{R} P^{2}\right) \longrightarrow S_{n}$ denote the natural epimorphism, and suppose that $\alpha$ is an element of $B_{n}\left(\mathbb{R} P^{2}\right)$ of order 2 different from $\Delta_{n}$. So $\alpha \notin P_{n}\left(\mathbb{R} P^{2}\right)$ by Proposition 15. It follows that the cycle decomposition of $\pi(\alpha)$ is a product of a non-zero number of transpositions and a certain number (perhaps zero) of cycles of length one. Let $D_{2, n-2}\left(\mathbb{R} P^{2}\right)=F_{n}\left(\mathbb{R} P^{2}\right) / S_{2} \times S_{n-2}$ be the intermediate covering between $F_{n}\left(\mathbb{R} P^{2}\right)$ and $D_{n}\left(\mathbb{R} P^{2}\right)$ GG2, and let $B_{2, n-2}\left(\mathbb{R} P^{2}\right)$ denote the corresponding subgroup of $B_{n}\left(\mathbb{R} P^{2}\right)$. Consider the homomorphism $\xi: B_{2, n-2}\left(\mathbb{R} P^{2}\right) \longrightarrow B_{2}\left(\mathbb{R} P^{2}\right)$ induced by the projection $D_{2, n-2}\left(\mathbb{R} P^{2}\right) \longrightarrow$ $D_{2}\left(\mathbb{R} P^{2}\right)$ onto the first two coordinates. By conjugating $\alpha$ if necessary, we may suppose without loss of generality that $\alpha \in B_{2, n-2}\left(\mathbb{R} P^{2}\right)$, and that the permutation of $\xi(\alpha)$ is a transposition. Since $\alpha$ is of order 2 then so is $\xi(\alpha)$. But from $\sqrt{\mathrm{VB}}$, the group $B_{2}\left(\mathbb{R} P^{2}\right)$ is known to be the dicyclic group of order 16 comprised of the identity, 10 elements of order 4,4 elements of order 8 
and a single element of order 2 which is the full twist $\Delta_{2}$. But this cannot be the case because $\Delta_{2} \in P_{2}\left(\mathbb{R} P^{2}\right)$, so its permutation is trivial. We thus obtain a contradiction, and this proves the result.

We now derive a necessary condition on the permutation associated with a torsion element.

Lemma 24 (Fundamental Lemma) Let $n \in \mathbb{N}$. Let $\alpha \in B_{n}\left(\mathbb{R} P^{2}\right) \backslash P_{n}\left(\mathbb{R} P^{2}\right)$ be a torsion element. If the permutation $\pi(\alpha)$ contains at least two cycles of length one then $\alpha \neq \Delta_{n}$ but $\alpha^{2}=\Delta_{n}$.

Proof Let $\alpha \in B_{n}\left(\mathbb{R} P^{2}\right) \backslash P_{n}\left(\mathbb{R} P^{2}\right.$ ) (so $n \geq 2$ ) be a torsion element of order $k$ such that the permutation $\pi(\alpha)$ contains at least two cycles of length one. Since $\alpha \notin P_{n}\left(\mathbb{R} P^{2}\right)$, Proposition [15] implies that $\alpha \neq \Delta_{n}$. Now $\Delta_{n}$ belongs to $Z\left(B_{n}\left(\mathbb{R} P^{2}\right)\right)$, so $\alpha$ would satisfy the equation $\alpha^{2}=\Delta_{n}$ if and only if its conjugates did too. Conjugating $\alpha$ if necessary, we may thus assume without loss of generality that $\alpha$ belongs to the subgroup $B_{1,1, n-2}\left(\mathbb{R} P^{2}\right)$ of $B_{n}\left(\mathbb{R} P^{2}\right)$ corresponding to the intermediate covering $D_{1,1, n-2}\left(\mathbb{R} P^{2}\right)=F_{n}\left(\mathbb{R} P^{2}\right) / S_{1} \times S_{1} \times$ $S_{n-2}$. Consider the homomorphism $\xi: B_{1,1, n-2}\left(\mathbb{R} P^{2}\right) \longrightarrow P_{2}\left(\mathbb{R} P^{2}\right)$ induced by the projection $D_{1,1, n-2}\left(\mathbb{R} P^{2}\right) \longrightarrow F_{2}\left(\mathbb{R} P^{2}\right)$ onto the first two coordinates. Now $P_{2}\left(\mathbb{R} P^{2}\right) \cong Q_{8}$, thus $(\xi(\alpha))^{2}=\Delta_{2}^{\varepsilon}$, where $\varepsilon \in\{0,1\}$. Since $\Delta_{n} \in$ $B_{1,1, n-2}\left(\mathbb{R} P^{2}\right)$ and $\xi\left(\Delta_{n}\right)=\Delta_{2}$, we have that $\alpha^{2} \cdot \Delta_{n}^{-\varepsilon} \in \operatorname{Ker}(\xi)=B_{n-2}\left(\mathbb{R} P^{2} \backslash\right.$ $\left.\left\{x_{1}, x_{2}\right\}\right)$ which we know to be torsion free. But $\left(\alpha^{2} \cdot \Delta_{n}^{-\varepsilon}\right)^{2 k}=1$ because $\Delta_{n}$ is central in $B_{n}\left(\mathbb{R} P^{2}\right)$. Hence $\alpha^{2}=\Delta_{n}^{\varepsilon}$. If $\varepsilon=0$ then $\alpha^{2}=1$ which since $\alpha \neq 1$ implies that $\alpha=\Delta_{n}$ by Proposition [23. But we have already established that this cannot be the case. Hence $\varepsilon=1$, and thus $\alpha^{2}=\Delta_{n}$ as required.

Corollary 25 Let $n \in \mathbb{N}$. If $\alpha$ is a torsion element of $B_{n}\left(\mathbb{R} P^{2}\right)$ then its order divides $4 n$ or $4(n-1)$.

Proof Let $\ell$ denote the order of $\alpha$. If $\alpha \in P_{n}\left(\mathbb{R} P^{2}\right)$ then the result follows from Proposition 15. So suppose that the cycle decomposition of $\pi(\alpha)$ contains at least one cycle of length greater than or equal to two. Then there are three possibilities:

(1) the non-trivial cycles in the cycle decomposition of $\pi(\alpha)$ are all of the same length $\ell_{1}$. Set $\ell_{0}=1$, and for $i=0,1$, let $k_{i}$ be the number of cycles of length $\ell_{i}$ in the cycle decomposition of $\pi(\alpha)$. If $k_{0} \geq 2$ then it follows from the Fundamental Lemma that $\alpha^{2}=\Delta_{n}$, and hence $\alpha$ is of order 4 which divides $4 n$ as required. So suppose that $k_{0} \in\{0,1\}$. 
Then $\alpha^{\ell_{1}} \in P_{n}\left(\mathbb{R} P^{2}\right)$, and so by Corollary 14, either $\alpha^{\ell_{1}}$ is the identity element, and so $\alpha$ is of order $\ell$, or $\alpha^{\ell_{1}}$ is of order 2 , and so $\alpha$ is of order $2 \ell$, or else $\alpha^{\ell_{1}}$ is of order 4 , and so $\alpha$ is of order $4 \ell$ (we have used the fact that $\alpha^{j} \notin P_{n}\left(\mathbb{R} P^{2}\right)$ for all $\left.1 \leq j<\ell\right)$. Since $n=k_{0}+\ell_{1} k_{1}$, we see that $\ell_{1}$ divides $n$ or $n-1$, and so the order of $\alpha$ divides $4 n$ or $4(n-1)$ as required.

(2) the non-trivial cycles in the cycle decomposition of $\pi(\alpha)$ are of two different lengths $\ell_{1}, \ell_{2}$ with $1<\ell_{1}<\ell_{2}$. Set $\ell_{0}=1$, and for $i=0,1,2$, let $k_{i}$ be the number of cycles of length $\ell_{i}$ in the cycle decomposition of $\pi(\alpha)$. Applying the Fundamental Lemma to $\alpha$, we see that $k_{0} \in\{0,1\}$. Consider the element $\alpha^{\ell_{1}}$; it belongs to $B_{n}\left(\mathbb{R} P^{2}\right) \backslash P_{n}\left(\mathbb{R} P^{2}\right)$, and contains at least two cycles of length one. Applying the Fundamental Lemma, it follows that $\ell_{2}=2 \ell_{1}$, and $\alpha^{2 \ell_{1}}=\Delta_{n}$. Hence $\alpha$ is of order $4 \ell_{1}$. Now $n=k_{0}+k_{1} \ell_{1}+k_{2} \ell_{2}=k_{0}+k_{1} \ell_{1}+2 k_{2} \ell_{1}$, hence $\ell_{1}$ divides $n$ or $n-1$, and thus the order $4 \ell_{1}$ of $\alpha$ divides $4 n$ or $4(n-1)$ as required.

(3) the cycle decomposition of $\pi(\alpha)$ contains cycles of at least three different lengths $\ell_{1}, \ell_{2}, \ell_{3}$ with $1<\ell_{1}<\ell_{2}<\ell_{3}$. Then $\alpha^{\ell_{1}}$ is a torsion element belonging to $B_{n}\left(\mathbb{R} P^{2}\right) \backslash P_{n}\left(\mathbb{R} P^{2}\right)$ containing at least two cycles of length one. By the Fundamental Lemma, $\alpha^{2 \ell_{1}}=\Delta_{n}$, so the cycle decomposition of $\pi(\alpha)$ contains only transpositions and trivial cycles, but this is impossible since $\ell_{1}<\ell_{2}<\ell_{3}$.

Let $n \geq 2$. We will show that there are elements in $B_{n}\left(\mathbb{R} P^{2}\right)$ of order $4 n$ and of order $4(n-1)$. In $B_{n}\left(\mathbb{R} P^{2}\right)$, consider the following elements:

$$
\left.\begin{array}{l}
a=\sigma_{n-1}^{-1} \cdots \sigma_{1}^{-1} \cdot \rho_{1} \\
b=\sigma_{n-2}^{-1} \cdots \sigma_{1}^{-1} \cdot \rho_{1} .
\end{array}\right\}
$$

Proposition 26 Let $n \geq 2$. In $B_{n}\left(\mathbb{R} P^{2}\right)$, $a$ is of order $4 n$ and $b$ is of order $4(n-1)$.

Proof We start by proving that $a^{2 n}=\Delta_{n}$. As in Proposition 18, we shall do this by showing that $a^{2 n}$ is a non-trivial element of $B_{n}\left(\mathbb{R} P^{2}\right)$ that commutes with each of the generators $\sigma_{1}, \ldots, \sigma_{n-1}, \rho_{1}$. We first establish two useful identities. Using Proposition [16 and equations (4) and (7), we have that:

$$
\begin{aligned}
\sigma_{n-1}^{-1} \cdots \sigma_{1}^{-1} \rho_{1} \sigma_{1} \cdots \sigma_{n-1} & =\sigma_{n-1}^{-1} \cdots \sigma_{1}^{-1} \rho_{1} \sigma_{1}^{-1} \cdots \sigma_{n-1}^{-1} \sigma_{n-1} \cdots \sigma_{2} \sigma_{1}^{2} \sigma_{2} \cdots \sigma_{n-1} \\
& =\rho_{n} \cdot \rho_{n}^{-2}=\rho_{n}^{-1},
\end{aligned}
$$

and

$$
\sigma_{1} \sigma_{2} \cdots \sigma_{n-1} \rho_{1} \sigma_{n-1}^{-1} \cdots \sigma_{1}^{-1}=\sigma_{1} \rho_{1} \sigma_{1}^{-1}=\sigma_{1}^{2} \rho_{2} .
$$


Using Proposition [16] and induction, we see that $a^{-i} \sigma_{1} a^{i}=\sigma_{i+1}$ for all $0 \leq i \leq$ $n-2$. Further, writing $a_{1}=\sigma_{n-1}^{-1} \cdots \sigma_{1}^{-1}$, we see that

$$
a^{-1} \sigma_{n-1} a=\rho_{1}^{-1} \sigma_{1} \cdots \sigma_{n-2} \sigma_{n-1} \sigma_{n-1} \sigma_{n-1}^{-1} \sigma_{n-2}^{-1} \cdots \sigma_{i}^{-1} \rho_{1},
$$

and applying the braid relations, it follows that $a^{-1} \sigma_{n-1} a=\rho_{1}^{-1} a_{1} \sigma_{1} a_{1}^{-1} \rho_{1}$. From this, we obtain $a^{-1}\left(a^{-1} \sigma_{n-1} a\right) a=\left(a^{-1} \rho_{1}^{-1} a_{1} a\right) \sigma_{2}\left(a^{-1} \rho_{1}^{-1} a_{1} a\right)^{-1}$. Equations (12) and (13) yield:

$$
\begin{aligned}
a^{-1} \rho_{1}^{-1} a_{1} a & =\rho_{1}^{-1} \sigma_{1} \cdots \sigma_{n-1} \rho_{1}^{-1} \sigma_{n-1}^{-1} \cdots \sigma_{1}^{-1} \sigma_{n-1}^{-1} \cdots \sigma_{1}^{-1} \rho_{1} \\
& =\rho_{1}^{-1} \rho_{2}^{-1} \sigma_{1}^{-2} \rho_{n}^{-1} \sigma_{n-1}^{-1} \cdots \sigma_{1}^{-1} .
\end{aligned}
$$

Since $\sigma_{n-1}^{-1} \cdots \sigma_{1}^{-1} \sigma_{2} \sigma_{1} \cdots \sigma_{n-1}=\sigma_{1}$, we obtain

$$
\begin{aligned}
a^{-2} \sigma_{n-1} a^{2} & =\rho_{1}^{-1} \cdot \rho_{2}^{-1} \sigma_{1}^{-2} \cdot \rho_{n}^{-1} \sigma_{n-1}^{-1} \cdots \sigma_{1}^{-1} \cdot \sigma_{2} \cdot \sigma_{1} \cdots \sigma_{n-1} \rho_{n} \sigma_{1}^{2} \rho_{2} \rho_{1} \\
& =\rho_{1}^{-1} \rho_{2}^{-1} \sigma_{1} \rho_{2} \rho_{1} .
\end{aligned}
$$

Finally, by equation (5), we see that

$$
a^{-1}\left(a^{-1} \sigma_{n-1} a\right) a=\rho_{1}^{-1} \rho_{2}^{-1} \sigma_{1} \rho_{2} \rho_{1}=\rho_{1}^{-1} \cdots \rho_{n}^{-1} \sigma_{1} \rho_{n} \cdots \rho_{1}=\sigma_{1}^{-1} .
$$

Thus the conjugates of $\sigma_{1}$ by successive powers of $a^{-1}$ are:

$$
\sigma_{1}, \ldots, \sigma_{n-1}, a^{-1} \sigma_{n-1} a, \sigma_{1}^{-1}, \ldots, \sigma_{n-1}^{-1}, a^{-1} \sigma_{n-1}^{-1} a,
$$

and then $\sigma_{1}$ after $2 n$ conjugations. In particular, $a^{-2 n} \sigma_{i} a^{2 n}=\sigma_{i}$ for all $1 \leq$ $i \leq n-1$, and so $a^{2 n}$ commutes with $\sigma_{i}$.

Now consider the successive conjugates of $\rho_{1}$ by $a^{-1}$. Using Proposition 16] and induction, we see that $a^{-i} \rho_{1} a^{i}=\rho_{i+1}$ for all $0 \leq i \leq n-1$. By equations (4) and (7), we have that

$$
\rho_{n}=\sigma_{n-1}^{-1} \cdots \sigma_{1}^{-1} \rho_{1} \sigma_{1}^{-1} \cdots \sigma_{n-1}^{-1}=a \rho_{1} a^{-1} \rho_{n}^{2} .
$$

It follows from this that $a^{-1} \rho_{n} a=\rho_{1}^{-1}$, and hence that the successive conjugates of $\rho_{1}$ by $a^{-1}$ are $\rho_{1}, \ldots \rho_{n}, \rho_{1}^{-1}, \ldots, \rho_{n}^{-1}$ and then $\rho_{1}$. So $a^{-2 n} \rho_{1} a^{2 n}=\rho_{1}$, and with the conclusion of the previous paragraph, we see that $a^{2 n}$ is central in $B_{n}\left(\mathbb{R} P^{2}\right)$.

Suppose that $a^{2 n}=1$. Since the permutation associated with $a$ is an $n$-cycle, it follows that $a$ is of order $n$ or $2 n$, and so $a^{n}$ is central. If $a$ is of order $n$ this is obvious, while if $a$ is of order $2 n$, we have that $\left(a^{n}\right)^{2}=1$, and so $a^{n}=\Delta_{n}$ by Proposition [15] But $a^{-n} \sigma_{1} a^{n}=\sigma_{1}^{-1}$ from above, which yields a contradiction. Since $Z\left(B_{n}\left(\mathbb{R} P^{2}\right)\right)$ is generated by the full twist $\Delta_{n}$ of order 2 , we deduce that $a^{2 n}=\Delta_{n}$, and since $n$ divides the order of $a$, it follows that $a$ is of order $4 n$. 
Now let us show that $b^{2(n-1)}=\Delta_{n}$. Notice first that $b=\sigma_{n-1} a$. By induction, we see that for all $1 \leq j \leq n-1, b^{j}=a^{j} \sigma_{j-1}^{-1} \cdots \sigma_{1}^{-1} \cdot a^{-1} \sigma_{n-1} a$. Indeed if the result is true for $1 \leq j \leq n-2$ then using the conclusion of the first paragraph of this proof, we see that

$$
\begin{aligned}
b^{j+1} & =a^{j} \sigma_{j-1}^{-1} \cdots \sigma_{1}^{-1} \cdot a^{-1} \sigma_{n-1} a \cdot \sigma_{n-1} a \\
& =a^{j+1} a^{-1} \sigma_{j-1}^{-1} \cdots \sigma_{1}^{-1} \cdot a a^{-2} \sigma_{n-1} a^{2} \cdot a^{-1} \sigma_{n-1} a \\
& =a^{j+1} \sigma_{j}^{-1} \cdots \sigma_{1}^{-1} a^{-1} \sigma_{n-1} a .
\end{aligned}
$$

Hence

$$
\begin{aligned}
b^{2(n-1)} & =\left(a^{n-1} \sigma_{n-2}^{-1} \cdots \sigma_{1}^{-1} a^{-1} \sigma_{n-1} a\right)^{2} \\
& =a^{2 n-(n+1)}\left(\sigma_{n-2}^{-1} \cdots \sigma_{1}^{-1} a^{-1} \sigma_{n-1} a\right) a^{n+1-2}\left(\sigma_{n-2}^{-1} \cdots \sigma_{1}^{-1} a^{-1} \sigma_{n-1} a\right) a a^{-1} \\
& =\Delta_{n} \sigma_{n-1} \cdots \sigma_{1} a^{-1} \sigma_{n-1}^{-1} \cdots \sigma_{1}^{-1} a^{-1} \\
& =\Delta_{n} \sigma_{n-1} \cdots \sigma_{1} \rho_{1}^{-2} \sigma_{1} \cdots \sigma_{n-1}=\Delta_{n},
\end{aligned}
$$

by Proposition 16. As for the case of $a$, to see that $b$ is of order $4(n-1)$, it suffices to prove that $b^{n-1}$ is not central. An easy induction argument shows that $b^{-i} \sigma_{1} b^{i}=\sigma_{i+1}$ for $0 \leq i \leq n-3$. Further, a calculation analogous to that above of $a^{-2} \sigma_{n-1} a^{2}$ shows that $b^{-2} \sigma_{n-1} b^{2}=\sigma_{1}^{-1}$. So $b^{-(n-1)} \sigma_{1} b^{(n-1)}=\sigma_{1}^{-1}$, thus $b^{n-1}$ is not central, and the proof of the proposition is complete.

This enables us to prove Theorem 4

Proof of Theorem 4 To prove the 'only if' part, it suffices to consider an appropriate power of one of the elements $a$ and $b$, and to apply Proposition 26 The 'if' part follows directly from Corollary 25.

Remark 27 It is possible (by an induction argument for example) to prove that $b^{n-1}=\rho_{n-1} \cdots \rho_{1}$ and $a^{n}=\rho_{n} \cdots \rho_{1}$. Then the 'only if' part of Theorem 4 also follows from Propositions 18 and 20 .

Finally, we are able to prove Theorem 5

Proof of Theorem 5 To prove the 'only if' part, let $\alpha$ be a $k^{\text {th }}$ root of the full twist. Then $\alpha$ is a torsion element of $B_{n}\left(\mathbb{R} P^{2}\right)$ of order $2 k$. By Theorem 4 it follows that $2 k$ divides either $4 n$ or $4(n-1)$, and the result follows. To prove the 'if part', the element $a$ (respectively, $b$ ) given in equation (11) has the property that its $2 n^{\text {th }}$ (respectively, $2(n-1)^{\text {th }}$ ) power is of order 2 , and so is the full twist $\Delta_{n}$ by Proposition 23 . The result then follows by taking an appropriate power of $a$ or $b$. 
Remark 28 In the case $n=3$, it follows from Remark 22 that our results in this section are compatible with those of Murasugi.

\section{References}

[A1] E Artin, Theorie der Zöpfe, Abh. Math. Sem. Univ. Hamburg 4 (1925) 47-72.

[A2] E Artin, Theory of braids, Ann. Math. 48 (1947) 101-126. MathReview

[A3] E Artin, Braids and permutations, Ann. Math. 48 (1947) 643-649. MathReview

[Ba] H J Baues, Obstruction theory on homotopy classification of maps, Lecture Notes in Mathematics 628, Springer-Verlag, Berlin, 1977. MathReview

[Bi] J S Birman, On braid groups, Comm. Pure and Appl. Math. 22 (1969) 41-72. MathReview

[CG] F R Cohen, S Gitler, On loop spaces of configuration spaces, Trans. Amer. Math. Soc. 354 (2002) 1705-1748. MathReview

[CJ] M Crabb, I James, Fibrewise homotopy theory, Springer monographs in mathematics, Springer-Verlag, London, 1998. MathReview

[Fa] E Fadell, Homotopy groups of configuration spaces and the string problem of Dirac, Duke Math. Journal 29 (1962) 231-242. MathReview

[FaN] E Fadell, L Neuwirth, Configuration spaces, Math. Scand. 10 (1962) 111-118. MathReview

[FVB] E Fadell, J Van Buskirk, The braid groups of $\mathbb{E}^{2}$ and $\mathbb{S}^{2}$, Duke Math. Journal 29 (1962) 243-257. MathReview

[FoN] R H Fox, L Neuwirth, The braid groups, Math. Scand. 10 (1962) 119-126. MathReview

[GG1] D L Gonçalves, J Guaschi, On the structure of surface pure braid groups, J. Pure Appl. Algebra 182 (2003) 33-64 (due to a printer's error, this article was republished in its entirety with the reference 186 (2004) 187-218). MathReview

[GG2] D L Gonçalves, J Guaschi, The roots of the full twist for surface braid groups, Math. Proc. Camb. Phil. Soc. 137 (2004) 307-320.

[GG3] D L Gonçalves, J Guaschi, On the structure of pure braid groups for nonorientable surfaces, in preparation.

[Hi] MW Hirsch, Differential topology, Graduate Texts in Mathematics 33, Corrected reprint of the 1976 original, Springer-Verlag, New York, 1994. MathReview

$[\mathrm{J}] \quad$ D L Johnson, Presentation of groups, LMS Lecture Notes 22 (1976), Cambridge University Press. MathReview 
[M] K Murasugi, Seifert fibre spaces and braid groups, Proc. London Math. Soc. 44 (1982) 71-84. MathReview

[PR] L Paris, D Rolfsen, Geometric subgroups of surface braid groups, Ann. Inst. Fourier (Grenoble) 49 (1999) 417-472. MathReview

[S] G P Scott, Braid groups and the group of homeomorphisms of a surface, Proc. Camb. Phil. Soc. 68 (1970) 605-617.

[VB] J Van Buskirk, Braid groups of compact 2 -manifolds with elements of finite order, Trans. Amer. Math. Soc. 122 (1966) 81-97. MathReview

[Wa] J H Wang, On the braid groups for $\mathbb{R} P^{2}$, J. Pure Appl. Algebra 166 (2002) 203-227. MathReview

[Wh1] G W Whitehead, Elements of homotopy theory, Graduate Texts in Mathematics 61, Springer-Verlag, New York, 1978. MathReview

[Wh2] J H C Whitehead, Combinatorial homotopy I, Bull. Amer. Math. Soc. 55 (1949) 213-245. MathReview

Departamento de Matemática - IME-USP

Caixa Postal 66281 - Ag. Cidade de São Paulo

CEP: 05311-970 - São Paulo - SP - Brasil

and

Laboratoire de Mathématiques Emile Picard, UMR CNRS 5580 UFR-MIG

Université Toulouse III, 118, route de Narbonne

31062 Toulouse Cedex 4, France

Email: dlgoncal@ime.usp.br and guaschi@picard.ups-tlse.fr

Received: 11 December 2003 